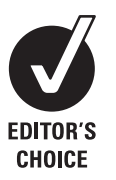
p 248

${ }^{1}$ Department of Neurology, Karolinska University Hospital, Stockholm, Sweden; ${ }^{2}$ Division of Clinical Neurosciences, University of Edinburgh, Edinburgh, UK; ${ }^{3}$ Department of Neuroradiology, University Hospital, University of Technology, Dresden, Germany; ${ }^{4}$ Department of Neurology, Haukeland University Hospital, Bergen, Norway

\section{Correspondence to:}

Dr T Kharitonova, SITS

International Coordination Office, Karolinska Stroke Research Unit, Department of Neurology, Karolinska University HospitalSolna, SE-171 76 Stockholm, Sweden; tatiana.kharitonova@ ki.se

Received 25 March 2008 Revised 6 July 2008 Accepted 16 July 2008 Published Online First 17 October 2008

\title{
Disappearing hyperdense middle cerebral artery sign in ischaemic stroke patients treated with intravenous thrombolysis: clinical course and prognostic significance
}

\author{
T Kharitonova, ${ }^{1}$ M Thorén, ${ }^{1} \mathrm{~N}$ Ahmed, ${ }^{1} \mathrm{~J}$ M Wardlaw, ${ }^{2} \mathrm{R}$ von Kummer, ${ }^{3} \mathrm{~L}$ Thomassen, ${ }^{4}$ \\ N Wahlgren, ${ }^{1}$ for the SITS investigators
}

\begin{abstract}
Background and purpose: Hyperdense middle cerebral artery sign (HMCAS) on CT is a well known indication of thromboembolic arterial occlusion. Its disappearance after thrombolytic therapy is poorly described. Taking the rate of HMCAS disappearance as a surrogate for MCA recanalisation, its prognostic value after intravenous thrombolysis was examined.
\end{abstract}

Methods: 1905 stroke patients with HMCAS on admission CT scan in the Safe Implementation of Treatment in Stroke-International Stroke Thrombolysis Register (SITS-ISTR) were studied. On follow-up CT scans 22-36 h after thrombolysis, HMCAS disappeared in 831 cases, persisted in 788 and was uncertain in 122; followup CT was not done in 164 cases.

Results: Patients whose HMCAS disappeared were younger (median age 67 years vs 69 years for persistent; $p=0.03$ ), with milder stroke (admission National Institute of Health Stroke Scale (NIHSS) score was 16 vs 17; $\mathrm{p}<0.005)$ and were less likely to have early infarct signs on admission CT ( $26 \%$ vs $33 \%$; $p<0.005)$. Patients with disappearing HMCAS were more likely to have early improvement in NIHSS score (median improvement 2 vs 0 at 2 h; 4 vs 1 at 24 h), be independent at 3 months (42\% vs $19 \%)$, with fewer deaths (15\% vs $30 \%$ ) than those with persistent HMCAS. In multivariate analysis, HMCAS disappearance independently predicted functional independence and survival. Early NIHSS improvement independently predicted HMCAS disappearance.

Conclusions: HMCAS disappeared after intravenous thrombolysis in about half of cases and these patients had twice as good outcomes compared with those with persistent HMCAS. The prognosis in patients with MCA occlusion that persists after intravenous thrombolysis is poor, which may indicate the need for an alternative treatment approach to this subgroup.

In acute ischaemic stroke, hyperdense middle cerebral artery sign (HMCAS) on admission CT scan is a known indication of middle cerebral artery occlusion. ${ }^{1-5}$ Its disappearance after thrombolytic therapy implies vessel recanalisation, as verified by cerebral angiography in observational studies. ${ }^{126}$ HMCAS disappearance on follow-up CT scan may be associated with better outcome. ${ }^{7}$ HMCAS on admission CT is associated with other early infarct signs, ${ }^{89}$ poor prognosis in patients who do not receive thrombolysis ${ }^{10}$ as well as in patients undergoing intravenous thrombolysis, ${ }^{11-13}$ and with higher probability of haemorrhagic transformation $^{14}{ }^{15}$ after intravenous thrombolysis, while intra-arterial thrombolysis demonstrates more encouraging outcomes in non-randomised studies. ${ }^{16-18}$ There are limited data on the rate of disappearance of HMCAS after intravenous thrombolysis, and its association with neurological and functional outcome. Information on intravenous thrombolysis in patients with HMCAS would be useful as the effectiveness of intravenous thrombolysis in these patients has been questioned and it is uncertain whether intra-arterial interventions should be a firstline therapy. ${ }^{18}$ Alternatively, intraarterial interventions such as thrombectomy could be considered for patients with failed intravenous thrombolysis. The aim of this study was to examine the rate of HMCAS disappearance and its prognostic value after intravenous thrombolysis.

\section{METHODS}

The study cohort consisted of 1905 stroke patients with HMCAS on admission CT scan who were registered in the Safe Implementation of Treatment in Stroke-International Stroke Thrombolysis Register (SITS-ISTR) database. SITS-ISTR is an international ongoing internet based open database of stroke thrombolytic treatment. For this study we used the data collected prospectively between December 2002 and October 2006. The structure of the database and the main results from the observational Safe Implementation of Thrombolysis in StrokeMOntoring STudy (SITS-MOST) were reported previously. ${ }^{19}$ Ethics approval was provided in each participating country in accordance with national legislation. All patients registered were treated with intravenous alteplase (Actilyse, Boehringer Ingelheim $\mathrm{GmbH}$, Germany). The study protocol was based on standard guidelines for intravenous thrombolysis, with pretreatment CT to exclude haemorrhagic stroke and stroke mimics and initiation of treatment within $3 \mathrm{~h}$ of stroke onset. The register contains baseline data, results of follow-up neurological assessment and relevant findings of CT scans, inhospital treatment and 3 month modified Rankin score. Baseline records included chronology (time of stroke onset, admission, imaging, start of treatment), demographic data, patient's weight, stroke risk factors (hypertension, hyperlipidaemia, smoking, atrial fibrillation, congestive heart failure, history of previous stroke, 
Table 1 Baseline data for the HMCAS disappearance and HMCAS persistence subgroups

\begin{tabular}{|c|c|c|c|}
\hline & $\begin{array}{l}\text { HMCAS-D } \\
(\mathrm{n}=\mathbf{8 3 1})\end{array}$ & $\begin{array}{l}\text { HMCAS-P } \\
(\mathrm{n}=788)\end{array}$ & p Value \\
\hline Age (years) (median (IQR)) & $67(58-75)$ & $69(59-76)$ & 0.03 \\
\hline Men (n (\%)) & $467(56.2)$ & $466(59.1)$ & 0.3 \\
\hline Weight $(\mathrm{kg})$ (mean (SD)) & $76.6(13.8)$ & $77.0(14.5)$ & 0.6 \\
\hline \multicolumn{4}{|l|}{ Stroke risk factors } \\
\hline No of risk factors (median (IQR)) & $2(1-2)$ & $2(1-2)$ & 0.7 \\
\hline Hypertension (n (\%)) & $468(56.3)$ & $478(60.7)$ & 0.2 \\
\hline Diabetes (n (\%)) & $116(14.0)$ & $114(14.5)$ & 0.9 \\
\hline Hyperlipidaemia (n (\%)) & $253(30.5)$ & $245(31.1)$ & 0.8 \\
\hline Smoking, current and previous (n (\%)) & $326(39.3)$ & $289(36.7)$ & 0.3 \\
\hline History of stroke (n (\%)) & $84(10.1)$ & $66(8.4)$ & 0.2 \\
\hline Atrial fibrillation (n (\%)) & $229(27.6)$ & $234(29.7)$ & 0.6 \\
\hline Congestive heart failure $(\mathrm{n}(\%))$ & $66(8.0)$ & $59(7.5)$ & 0.4 \\
\hline Pre-existing disability $=$ mRS $2-5$ at baseline $(\mathrm{n}(\%))$ & $54(6.5)$ & $63(8.0)$ & 0.5 \\
\hline \multicolumn{4}{|l|}{ Baseline treatment } \\
\hline Aspirin (n (\%)) & $234(28.2)$ & $226(28.7)$ & 0.8 \\
\hline Dipyridamol (n (\%)) & $8(1.0)$ & $10(1.3)$ & 0.7 \\
\hline Clopidogrel (n (\%)) & $33(4.0)$ & $26(3.3)$ & 0.9 \\
\hline Other antiplatelet drug $(\mathrm{n}(\%))$ & $10(1.2)$ & $12(1.5)$ & 0.5 \\
\hline Anticoagulant IV, therapeutic dose (n (\%)) & $4(0.5)$ & $3(0.4)$ & 0.6 \\
\hline Anticoagulant IV, prophylactic dose (n (\%)) & $23(2.8)$ & $14(1.8)$ & 0.1 \\
\hline Anticoagulant oral (n (\%)) & $13(1.6)$ & $9(1.1)$ & 0.4 \\
\hline Antihypertensive IV (n (\%)) & $42(5.1)$ & $49(6.2)$ & 0.2 \\
\hline Antihypertensive oral (n (\%)) & $341(40.1)$ & $365(46.3)$ & 0.01 \\
\hline \multicolumn{4}{|l|}{ Time period } \\
\hline Onset to treatment time (min) (median (IQR)) & $135(105-163)$ & $140(110-165)$ & 0.1 \\
\hline Onset to imaging time (min) (median (IOR))) & $89(67-115)$ & $90(67-120)$ & 0.2 \\
\hline Onset to hospital time (min) (median (IQR)) & $63(44-90)$ & $63(44-90)$ & 0.9 \\
\hline \multicolumn{4}{|l|}{ Admission data } \\
\hline Admission plasma cholesterol (mmol/l) (mean (SD)) & $7.8(3.4)$ & $7.9(3.4)$ & 0.7 \\
\hline Admission plasma glucose $(\mathrm{mmol} / \mathrm{l})$ (mean (SD)) & $7.1(3.1)$ & $7.1(2.4)$ & 0.9 \\
\hline NIHSS score on admission (median (IQR)) & $16(12-20)$ & $17(14-20)$ & $<0.001$ \\
\hline Systolic blood pressure (mm Hg) (mean (SD)) & $147.1(20.7)$ & $148.7(22.0)$ & 0.3 \\
\hline Diastolic blood pressure $(\mathrm{mm} \mathrm{Hg})$ (mean (SD)) & $81.6(12.7)$ & $80.9(13.4)$ & 0.4 \\
\hline Presence of infarct on admission CT/MRI scan (n (\%)) & $216(26.0)$ & $256(32.5)$ & 0.001 \\
\hline
\end{tabular}

HMCAS-D, hyperdense middle cerebral artery sign, disappearance; HMCAS-P, hyperdense middle cerebral artery sign, persistence; IV, intravenous; mRS, median Rankin Scale Score; NIHSS, National Institute of Health Stroke Scale.

pre-existing disability); pre-stroke medications (antiplatelet therapy, anticoagulants, antihypertensive treatment); admission blood pressure, plasma glucose and cholesterol levels; and results of neurological assessment and CT scanning. Neurological status was estimated by National Institute of Health Stroke Scale on admission and at 2 h, 24 h and on day 7.

Clinical subtypes of stroke were not available directly from the database. Nevertheless, in order to examine the rate of total anterior circulation infarct (TACI) syndrome, which is a clinical presentation of proximal MCA occlusion, it was derived as an approximation from the baseline National Institute of Health Stroke Scale (NIHSS) records. The syndrome was defined as a combination of higher cerebral dysfunction, homonymous visual field defect, and ipsilateral motor and/or sensory deficit of at least two areas of the face, arm and leg. ${ }^{20}$ Presence of TACI syndrome was set with the assumption that facial palsy and sensory deficit were ipsilateral to the motor deficit in the arm and leg.

Stroke aetiology was established in accordance with the TOAST classification. Admission CT scans were evaluated for the presence of HMCAS and ischaemic signs. Follow-up CT scans were performed within 22-36 h of stroke (or earlier if clinically indicated) and evaluated for the presence of HMCAS, ischaemic signs, infarct swelling and intracerebral haemorrhage.
Outcomes were independence (modified Rankin Score (mRS) 0 2 on day 90) and death ( $\mathrm{mRS}=6$ on day 90). Unfavourable outcome was defined as death or dependency (mRS 3-6). Symptomatic intracerebral haemorrhage (SICH) was defined as local or remote type 2 parenchymal haemorrhage on the $22-$ $36 \mathrm{~h}$ post-treatment imaging scan, accompanied by deterioration of baseline NIHSS score $\geqslant 4$ points or death within $24 \mathrm{~h}$ after thrombolysis (SITS-MOST definition). ${ }^{19}$ Additionally, we used a definition from previous randomised controlled trials: any haemorrhage on follow-up imaging combined with at least 1 point deterioration on NIHSS or death until day 7 . Neurological improvement was estimated by the difference between admission and follow-up NIHSS scores. Relation of extent of neurological improvement to outcome was estimated in the whole HMCAS group at several levels of NIHSS score difference. Dramatic neurological improvement was defined as NIHSS score 0,1 or improvement $\geqslant 8^{21}$ from baseline score at a follow-up time point.

HMCAS disappearance (HMCAS-D) and persistence (HMCAS-P) were identified on follow-up CT scans.

\section{Statistical analysis}

Baseline data, admission clinical variables, stroke aetiology, results of neurological assessment, other CT findings and 
Table 2 Clinical features and outcomes of HMCAS-disappearance (HMCAS-D) and HMCAS-persistence (HMCAS-P) subgroups

\begin{tabular}{|c|c|c|c|}
\hline & $\begin{array}{l}\text { HMCAS-D } \\
(\mathrm{n}=831)\end{array}$ & $\begin{array}{l}\text { HMCAS-P } \\
(\mathrm{n}=788)\end{array}$ & p Value \\
\hline \multicolumn{4}{|l|}{ TOAST subgroups (ICD-10 category) } \\
\hline Large vessel disease $(163.0+163.3)(\mathrm{n}(\%))$ & $328(41.3)$ & $319(46.2)$ & 0.06 \\
\hline Cardiac embolism (I63.4) (n (\%)) & $332(41.8)$ & $266(38.3)$ & 0.2 \\
\hline Small vessel disease (lacunar, I63.5) (n (\%)) & $9(1.1)$ & $6(0.9)$ & 0.7 \\
\hline \multicolumn{4}{|l|}{ Clinical course } \\
\hline NIHSS score at $2 \mathrm{~h}$ (median (IQR)) & $13(8-18)$ & $17(12-20)$ & $<0.001$ \\
\hline NIHSS score at $24 \mathrm{~h}$ (median (IOR)) & $11(4-17)$ & $17(12-21)$ & $<0.001$ \\
\hline NIHSS score at day 7 (median (IQR)) & $8(2-15)$ & $15(9-24)$ & $<0.001$ \\
\hline Neurological improvement $\geqslant 4$ points NIHSS at $2 \mathrm{~h}(\mathrm{n}(\%))$ & $274(35.1)$ & $123(17.1)$ & $<0.001$ \\
\hline Dramatic neurological improvement at $2 \mathrm{~h}(\mathrm{n}(\%))$ & $114(14.6)$ & $34(4.7)$ & $<0.001$ \\
\hline TACl syndrome (approximate definition) (n (\%)) & $397(47.7)$ & $411(52.2)$ & 0.08 \\
\hline Presence of infarct on follow-up CT scan (n (\%)) & $740(89.1)$ & $754(95.7)$ & $<0.001$ \\
\hline \multicolumn{4}{|l|}{ Outcomes } \\
\hline mRS at day 90 (median (IOR)) & $3(1-5)$ & $4(3-6)$ & $<0.001$ \\
\hline Independence (mRS 0-2 at day 90 ) (n (\%)) & $296(41.5)$ & $134(19.0)$ & $<0.001$ \\
\hline Mortality (n (\%)) & $111(15.3)$ & $214(30.2)$ & $<0.001$ \\
\hline SICH, SITS-MOST definition (\%) & 1.8 & 0.6 & 0.06 \\
\hline SICH, RCT definition (\%) & 8.5 & 12.3 & 0.02 \\
\hline
\end{tabular}

outcomes were compared between the HMCAS persistence and disappearance groups. Multivariate analyses were performed to identify prognostically important factors for HMCAS disappearance and to identify prognostic significance of HMCAS disappearance on main outcomes. Prognostic significance of HMCAS disappearance was adjusted for stroke risk factors and aetiology, onset to treatment time, admission NIHSS score, blood glucose and extent of immediate NIHSS improvement at $2 \mathrm{~h}$. Statistical analysis was performed with Statistica 6.0 software. Estimation of percentages was based on reported cases only, excluding missing data. For univariate comparison between HMCAS-D and HMCAS-P subgroups, we used the $t$ test for numerical data, the Mann-Whitney test for scores and non-parametrically distributed numerical variables, and the $\chi^{2}$ test (with Yates correction for $2 \times 2$ tables or, in case of small expected frequencies, Fisher's exact test) for proportions. For multivariate analysis, we performed multiple logistic regression to define independent predictors of functional outcome. Taking into account multiple comparisons, significance level for univariate testing was accepted as $\mathrm{p}<0.01$.

\section{RESULTS}

Of 1905 patients with HMCAS on admission CT scans, the sign disappeared in 831 (48\%), persisted in 788 (45\%) and was uncertain in $122(7 \%)$ on $22-36 \mathrm{~h}$ follow-up CT scans. In the remaining 164 cases, follow-up CT was omitted. The subgroup with uncertain HMCAS on follow-up CT scan demonstrated a trend to lower independence rate at 3 months $(24.6 \%$ vs $31.2 \%$; $p=0.1)$ and the same death rate $(23.4 \%$ vs $23.6 \% ; p=0.9)$ than the rest of the population of patients with HMCAS on admission CT scan $(n=1783)$. The subgroup with missing follow-up CT scans had a higher independence rate $(40.6 \%$ vs $29.8 \% ; p=0.01)$ and non-significantly more deaths (28\% vs $23 \%, p=0.2)$ than the rest of the population of patients with HMCAS on admission CT scan $(n=1741)$. We decided to exclude the missing and uncertain groups from further analyses, given the similarity of outcomes between these groups and the rest of the group of patients with admission HMCAS.

Results of univariate comparison of HMCAS-D and HMCASP subgroups are shown in tables 1 and 2. Virtually no difference was found regarding past medical history and chronology. In particular, the onset to imaging and treatment times were the same in both groups. Patients with HMCAS-D had a slightly lower median age, slightly lower admission NIHSS score and fewer ischaemic signs on admission CT. The proportions of patients with a TACI syndrome were approximately equal in subgroups with persistent and disappeared HMCAS on followup CT (no statistically significant difference, table 2).

The HMCAS-D subgroup had borderline three times higher rate of SICH according to the SITS-MOST definition compared with the HMCAS-P subgroup, but a lower rate of SICH according to the randomised controlled trials definition. Despite a higher rate of type 2 parenchymal haemorrhage, the HMCASD subgroup demonstrated a much more favourable NIHSS profile (fig 1). Twice as many achieved functional independence at 3 months with half as many deaths as in the HMCAS-P subgroup. Outcomes were approaching those for patients without HMCAS on the baseline scan (fig 1). Outcomes of non-HMCAS patients in the SITS register have been published elsewhere. $^{22}$ Early neurological improvement by $\geqslant 4$ points NIHSS at $2 \mathrm{~h}$ (post-treatment) was twice as common, and dramatic neurological improvement at $2 \mathrm{~h}$ occurred three times more often in HMCAS-D patients. Median change of NIHSS score from baseline in HMCAS-D and HMCAS-P patients was 2 versus 0 at $2 \mathrm{~h}$ and 4 versus 1 at $24 \mathrm{~h}(\mathrm{p}<0.001)$.

In our multivariate analysis, stroke risk factors, TOAST subgroups or admission blood glucose did not appear to predict good outcome. HMCAS disappearance was one of the strongest predictors of good functional outcome at 3 months (odds ratio 2.26 , 95\% confidence interval 1.68 to 3.05 ; $p<0.001$ ); other independent predictors of outcome in HMCAS patients were age, admission NIHSS score and extent of neurological improvement at $2 \mathrm{~h}$. Admission NIHSS score and extent of 

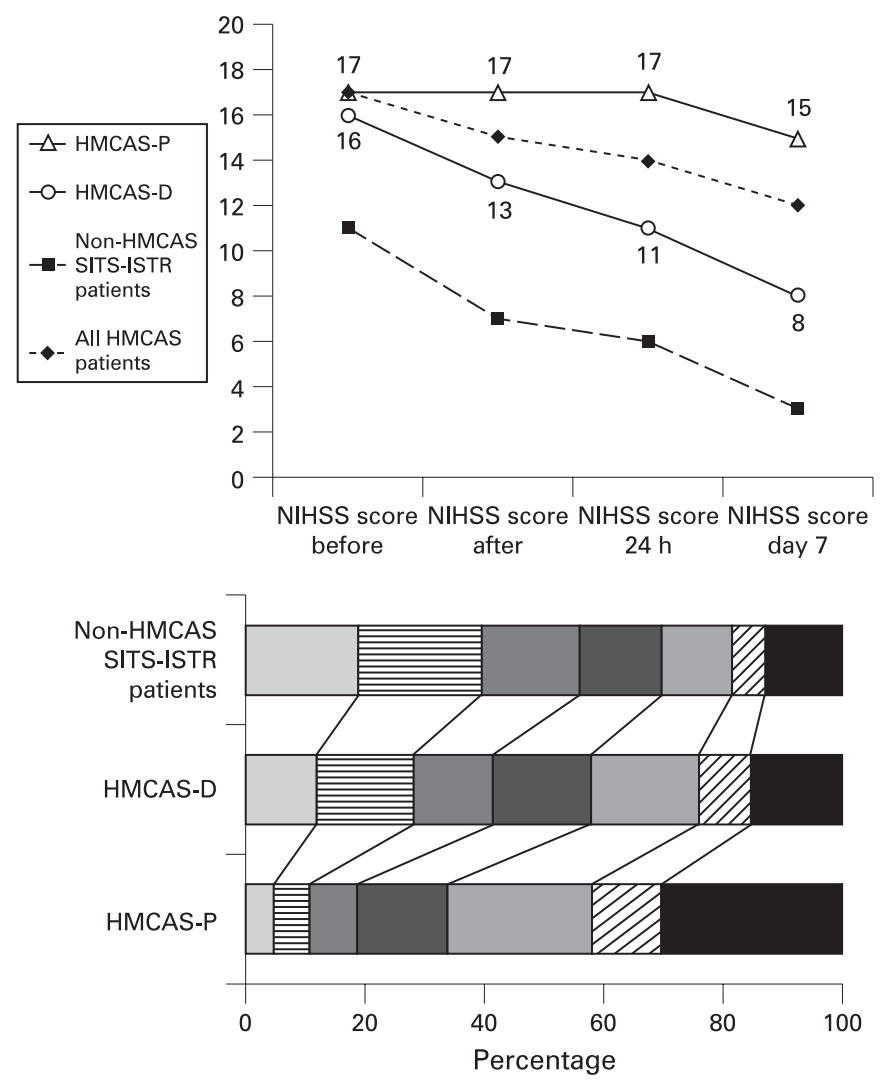

$\begin{array}{llll}\square \mathrm{mRS}=0 & \text { 目 } \mathrm{mRS}=1 & \square \mathrm{mRS}=2 & \square \mathrm{mRS}=3 \\ \mathrm{mRS}=4 & \square \mathrm{mRS}=5 & \mathrm{mRS}=6 & \end{array}$

Figure 1 NIHSS profiles and outcomes for patients with HMCAS-D, HMCAS-P, for all HMCAS patients and for SITS-ISTR patients without HMCAS on admission. HMCAS-D, hyperdense middle cerebral artery sign, disappearance; HMCAS-P, hyperdense middle cerebral artery sign, persistence; mRS, modified Rankin Scale Score; NIHSS, National Institute of Health Stroke Scale; P, persistence; SITS-ISTR, Safe Implementation of Thrombolysis in Stroke-International Stroke Thrombolysis Register.

neurological improvement at $2 \mathrm{~h}$ independently predicted HMCAS disappearance.

Based on the association between $2 \mathrm{~h}$ NIHSS improvement and 3 month outcome in patients with HMCAS disappearance, we categorised the $2 \mathrm{~h}$ NIHSS difference to identify a suitable cut-off level to be used clinically for prediction of outcomes

Table 3 Functional outcome at 3 months against change in NIHSS at $2 \mathrm{~h}$ in HMCAS-D and HMCAS-P subgroups

\begin{tabular}{|c|c|c|}
\hline \multirow{2}{*}{$\begin{array}{l}\text { Change in NIHSS score } \\
\text { at } 2 \mathrm{~h} \text { after baseline }\end{array}$} & \multicolumn{2}{|c|}{$\begin{array}{l}\text { Good functional outcome (mRS } 0-2 \text { at } \\
3 \text { months) }(\mathrm{n}(\%))(p=0.01)\end{array}$} \\
\hline & HMCAS-D & HMCAS-P \\
\hline$<0$ (i.e. worsening) & $20(27.4 \%)$ & $12(10.1 \%)$ \\
\hline$=0$ (no change) & $34(19.9 \%)$ & $29(12.9 \%)$ \\
\hline$=1$ (improvement) & $23(37.1 \%)$ & $6(7.1 \%)$ \\
\hline$=2$ & $28(40.6 \%)$ & $15(23.1 \%)$ \\
\hline$=3$ & $27(42.2 \%)$ & $17(40.5 \%)$ \\
\hline$=4$ & $20(43.5 \%)$ & $5(15.6 \%)$ \\
\hline$=5$ & $11(39.3 \%)$ & $10(50.0 \%)$ \\
\hline$>5$ & $115(71.4 \%)$ & $29(49.2 \%)$ \\
\hline
\end{tabular}

HMCAS-D, hyperdense middle cerebral artery sign, disappearance; HMCAS-P, hyperdense middle cerebral artery sign, persistence; $\mathrm{mRS}$, median Rankin Scale score; NIHSS, National Institute of Health Stroke Scale. (table 3, fig 2). The proportion of patients with favourable outcomes increased sequentially with each point NIHSS improvement at $2 \mathrm{~h}$. However, the most favourable outcomes were observed in those who demonstrated dramatic neurological improvement at $2 \mathrm{~h}: 75.2 \%(\mathrm{n}=94)$ were independent and $4.8 \%(\mathrm{n}=6)$ had died after 3 months compared with $25.7 \%$ (307) and 24.4\% (292), respectively, of those without dramatic improvement $(\mathrm{p}<0.001)$.

Repeated multivariate analyses were performed, including $2 \mathrm{~h}$ NIHSS improvement strata (decline in NIHSS score $\geqslant 4, \geqslant 3, \geqslant 2$ or $\geqslant 1$ from baseline) together with previously determined prognostically important factors (baseline NIHSS score, age and HMCAS disappearance). On testing various cut-off levels, we found that any improvement in NIHSS score from baseline (ie, difference between baseline and $2 \mathrm{~h}$ NIHSS scores of at least 1 ) was associated with good functional outcome.

\section{DISCUSSION}

To our knowledge, this is the largest study examining the rate of HMCAS disappearance and its prognostic value in patients treated with intravenous thrombolysis. HMCAS is a well known sign of MCA occlusion and a valuable, reliable and quick to assess prognostic sign in its own right. Its clinical use may become more limited following the development of multimodal imaging but its value when present should not be underestimated. However, inability to demonstrate details of occlusion, such as tandem or T-shaped clot, in occasional circumstances where this detail might be helpful, is the main weakness of plain CT in the setting of MCA stroke.

We observed that the HMCAS disappeared after intravenous thrombolysis on follow-up CT scan at 22-36 h after stroke onset in about half of patients. These patients showed more rapid neurological improvement and had better functional outcome, as measured by mRS $0-2$, and lower mortality at 3 months compared with patients in whom the HMCAS persisted. In the multivariate analysis of outcome predictors, disappearance of the HMCAS appeared to be one of the independent predictors of 3 month independence and reduced

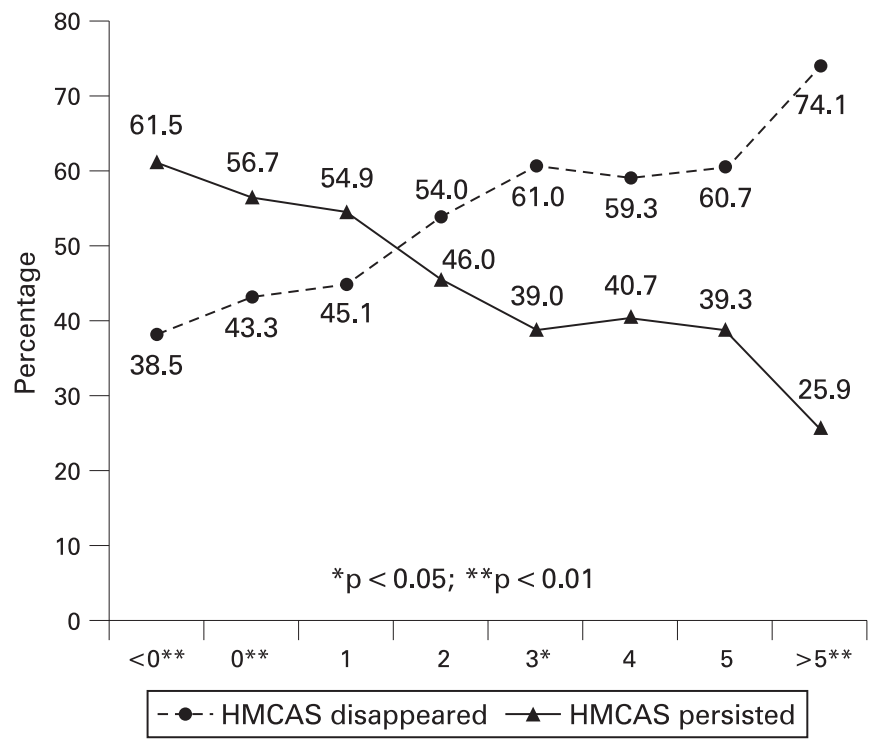

Figure 2 Proportions of HMCAS disappearance and persistence at the various levels of $2 \mathrm{~h}$ NIHSS change from baseline. HMCAS, hyperdense middle cerebral artery sign; NIHSS, National Institute of Health stroke scale. 
mortality. These results suggest that HMCAS-D patients are adequately recanalised as their outcomes were comparable with patients without the HMCAS on admission CT after adjustment for their initial stroke severity (fig 1). In contrast, HMCAS-P patients had very poor short and long term outcomes after intravenous thrombolysis. However, HMCAS disappearance after 22-36 h serves only as a landmark of large artery recanalisation and may be demonstrated too late to be a criterion for clinical decisions.

Some of the baseline parameters differed between the HMCAS-D and HMCAS-P subgroups but these differences cannot be used in clinical practice as prognostic markers for persistence because they were marginal. Patients with persistence were 2 years older, had 1 point more median baseline NIHSS and more often had signs of current infarction than those with disappearance, which may indicate that HMCAS-P patients had less favourable baseline data despite being imaged and treated at the same time after stroke. We hypothesised that the disappearance of HMCAS on follow-up CT scans indicated vessel recanalisation. The clinical course and outcome of the HMCAS-D subgroup support our hypothesis. When we followed the clinical course of all HMCAS patients, we considered whether absence of neurological improvement at $2 \mathrm{~h}$ might help to identify those in whom the HMCAS was likely to persist. We observed that HMCAS persisted in more than half of patients with any NIHSS improvement at $2 \mathrm{~h}$ (table 3 ). In the multivariate analysis, $2 \mathrm{~h}$ NIHSS changes, by as little as 1 point, appeared as one of the significant predictors for HMCAS disappearance but the overlap was substantial. In this setting, lack of neurological improvement cannot be the basis for the decision about additional intervention as this approach fails to identify almost half of patients with a poor prognosis in the HMCAS-P subgroup. Hence it seems reasonable to take patients with identified MCA occlusion and no clinical improvement after intravenous thrombolysis directly to the angiosuite in order to perform DSA and attempt mechanical recanalisation. This approach may be suggested for testing in a randomised controlled trial.

SICH, according to the SITS-MOST definition, was comparatively uncommon in the HMCAS-P subgroup while that of the HMCAS-D subgroup was close to the SICH rate observed in the SITS-MOST study. ${ }^{19}$ This finding may support the previously discussed hypothesis that recanalisation of an occluded vessel predisposes to subsequent haemorrhage..$^{152-25}$ The overall rate of severe ICH among HMCAS-D patients, which fall into SICH by the SITS-MOST category, was still small, hence it did not influence the generally favourable clinical course and proportion of good outcome in this subgroup. As for the higher prevalence of SICH by the randomised controlled trials definition in HMCAS-P patients, this observation may be partly explained by a large volume of necrotic infarction in patients with persistent MCA occlusion, which results in minor intracerebral haemorrhage.

Our results cannot provide an explanation for the initial small difference in baseline NIHSS scores between the HMCAS-D and HMCAS-P subgroups, suggesting that some other unrecorded factors may play a role, one of which could be, for instance, composition of the clot. ${ }^{26}$ We only recorded the presence of HMCAS, not its site or extent (it is possible that HMCAS-P patients had longer clots, or more proximal clots, that involved the ICA/proximal MCA, whereas HMCAS-D patients only had insular branch MCA clots, hence accounting for the difference in visible infarction and features suggesting more severe strokes in HMCAS-P patients). However, the observation that the difference in TACI syndromes between HMCAS-D and HMCAS-P subgroups was small and statistically insignificant speaks against this being a major explanation for the differences in outcome. The trend for more patients with large vessel disease in the HMCAS-P subgroup is in line with poorer outcomes after intravenous thrombolysis when MCA occlusion is combined with ICA obstruction. ${ }^{27}$ Moreover, one could speculate that these patients have a higher incidence of underlying arterial stenosis and subsequent re-occlusion after successful thrombolysis.

There are some limitations to our study. Firstly, this is an observational unblinded study. Secondly, approximately $12 \%$ of follow-up scans were missing. Thirdly, we did not have CT or $M R$ angiography or transcranial Doppler data for most of these patients (only $11 \%$ had CTA or MR angiography) to examine whether the disappearance of the sign correlated with vessel recanalisation, although that was not the aim of our study. Fourthly, follow-up CT scans were performed between 22 and $36 \mathrm{~h}$ after recombinant plasminogen activator treatment, as standard protocol. Therefore, we do not know how quickly the HMCAS disappeared, only what proportion had disappeared by 22-36 h. The scans were read by local doctors with wide variation in experience and expertise for early stroke CT assessment, so some unintentional errors could have occurred. It is possible that the NIHSS scores were influenced by knowledge of CT findings since the physicians were unblinded to the CT scans. Despite these limitations, this is the largest study on early follow-up of HMCAS patients given intravenous thrombolysis. Previous studies did not have more than a few hundred patients, ${ }^{2}{ }^{12} 13$ and in some cases less than a hundred patients were analysed, ${ }^{11}{ }^{124}$ although in these studies the scans were read centrally, usually by one expert, resulting in less potential noise in the HMCAS assessment.

\section{CONCLUSIONS}

The admission hyperdense MCA sign disappears on 22-36 h CT scans after intravenous thrombolysis in almost half of all cases. The proportion of functionally independent patients in the HMCAS disappearance subgroup was more than double and mortality was less than half compared with the HMCAS persistence subgroup. A higher prevalence of type 2 parenchymal haemorrhage in the HMCAS disappearance subgroup did not influence overall favourable 3 month outcome. The prognosis in patients with MCA occlusion that persists after intravenous thrombolysis is poor, which may indicate the appeal for alternative treatment approaches in this subgroup.

Acknowledgements: Scientific Committee of SITS International: Nils Wahlgren (chair), Antoni Davalos, Gary A Ford, Martin Grond, Werner Hacke, Michael Hennerici, Markku Kaste, Vincent Larrue, Kennedy R Lees, Risto Roine and Danilo Toni.

Funding: SITS-ISTR is funded by an unrestricted grant from Boehringer-Ingelheim (BI), and by a grant from the European Union Public Health Authority (PHEA). Preparation of this paper was independent of the funding organisation. The views expressed are those of the authors. Uppsala Clinical Research (UCR), Sweden, develops, maintains and upgrades the software for SITS register in close collaboration with SITS.

Competing interests: NA is an employee of SITS International. SITS International receives an unrestricted grant from Boehringer-Ingelheim. RvK has received honoraria for lectures and/or for advisory boards from Boehringer-Ingelheim, Sanofi-Aventis, AstraZeneca, Bayer, Pfizer, NovoNordisk, Astellas, Terumo, Paion and Novartis. JMW works for the University of Edinburgh and provided imaging expertise for the SITS-MOST registry, including design of the image data acquisition and reading of scans submitted to SITSMOST for expert review. She has received honoraria only for providing expert scan interpretation for SITS-MOST from SITS International who are supported by BoehringerIngelheim. LT has received honoraria for lectures and financial support for research nurses from Boehringer-Ingelheim. NW is chairman of SITS International. SITS International receives an unrestricted grant from Boehringer-Ingelheim. NW has received honoraria for 
lectures and/or for advisory boards from Boehringer-Ingelheim, Sanofi-Aventis, AstraZeneca, Bayer and Thrombogenics.

Ethics approval: Ethics approval was provided in each participating country in accordance with national legislation.

\section{REFERENCES}

1. Bastianello S, Pierallini A, Colonnese $C$, et al. Hyperdense middle cerebral artery CT sign. Comparison with angiography in the acute phase of ischemic supratentorial infarction. Neuroradiology 1991;33:207-11.

2. Leys D, Pruvo JP, Godefroy 0, er al. Prevalence and significance of hyperdense middle cerebral artery in acute stroke. Stroke 1992;23:317-24.

3. Wolpert SM, Bruckmann H, Greenlee R, et al. Neuroradiologic evaluation of patients with acute stroke treated with recombinant tissue plasminogen activator. The rt-PA Acute Stroke Study Group. AJNR Am J Neuroradiol 1993;14:3-13.

4. von Kummer R, Meyding-Lamade U, Forsting $M$, et al. Sensitivity and prognostic value of early CT in occlusion of the middle cerebral artery trunk. AJNR Am J Neuroradiol 1994:15:9-15.

5. Garg N, Eshkar N, Tanenbaum L, et al. Computed tomography angiographic correlates of early computed tomography signs in acute ischemic stroke. J Neuroimaging 2004;14:242-5.

6. Tsao JW, Gean AD, Glenn OA, et al. Hyperdense MCA resolved after tPA. Neurology 2002; 58:1512.

7. Muir KW, Buchan A, von Kummer R, et al. Imaging of acute stroke. Lancet Neurol 2006;5:755-68.

8. Moulin T, Cattin F, Crepin-Leblond T, et al. Early CT signs in acute middle cerebral artery infarction: predictive value for subsequent infarct locations and outcome. Neurology 1996:47:366-75

9. Koga M, Saku Y, Toyoda K, et al. Reappraisal of early CT signs to predict the arterial occlusion site in acute embolic stroke. J Neurol Neurosurg Psychiatry 2003; 74:649-53.

10. Wardlaw JM, Mielke 0 . Early signs of brain infarction at CT: observer reliability and outcome after thrombolytic treatment-systematic review. Radiology 2005;235:444-53.

11. Tomsick T, Brott T, Barsan W, et al. Prognostic value of the hyperdense middle cerebral artery sign and stroke scale score before ultraearly thrombolytic therapy. AJNR Am J Neuroradiol 1996;17:79-85.

12. Manelfe C, Larrue V, von Kummer R, et al. Association of hyperdense middle cerebral artery sign with clinical outcome in patients treated with tissue plasminogen activator. Stroke 1999;30:769-72.
13. Qureshi Al, Ezzeddine MA, Nasar A, et al. Is IV tissue plasminogen activator beneficial in patients with hyperdense artery sign? Neurology 2006;66:1171-4.

14. Derex L, Hermier M, Adeleine P, et al. Clinical and imaging predictors of intracerebral haemorrhage in stroke patients treated with intravenous tissue plasminogen activator. J Neurol Neurosurg Psychiatry 2005;76:70-5.

15. Khatri P, Wechsler LR, Broderick JP. Intracranial hemorrhage associated with revascularization therapies. Stroke 2007:38:431-40.

16. Mattle HP, Arnold M, Georgiadis D, et al. Comparison of intraarterial and intravenous thrombolysis for ischemic stroke with hyperdense middle cerebral artery sign. Stroke 2008;39:379-83.

17. Bendszus $\mathbf{M}$, Urbach $\mathrm{H}$, Ries $\mathrm{F}$, et al. Outcome after local intra-arterial fibrinolysis compared with the natural course of patients with a dense middle cerebral artery on early CT. Neuroradiology 1998;40:54-8.

18. Agarwal P, Kumar S, Hariharan S, et al. Hyperdense middle cerebral artery sign: can it be used to select intra-arterial versus intravenous thrombolysis in acute ischemic stroke? Cerebrovasc Dis 2004;17:182-90.

19. Wahlgren N, Ahmed N, Davalos A, et al. Thrombolysis with alteplase for acute ischaemic stroke in the Safe Implementation of Thrombolysis in Stroke-Monitoring Study (SITS-MOST): an observational study. Lancet 2007;369:275-82.

20. Bamford J, Sandercock P, Dennis M, et al. Classification and natural history of clinically identifiable subtypes of cerebral infarction. Lancet 1991;337:1521-6.

21. Brown DL, Johnston KC, Wagner DP, et al. Predicting major neurological improvement with intravenous recombinant tissue plasminogen activator treatment of stroke. Stroke 2004;35:147-50.

22. Kharitonova T, Thorén M, Ahmed N, et al. Disappearance of hyperdense middle cerebral artery sign (HMCAS) after i.v. thrombolysis - results from 1.737 patients in the SITS database. Cerebrovasc Dis 2007;23/Suppl 2, European Stroke Conference):38.

23. Fisher M, Adams RD. Observations on brain embolism with special reference to the mechanism of hemorrhagic infarction. J Neuropathol Exp Neurol 1951;10:92-4.

24. Tomsick TA, Brott TG, Olinger CP, et al. Hyperdense middle cerebral artery: incidence and quantitative significance. Neuroradiology 1989;31:312-15.

25. Molina CA, Alvarez-Sabin J, Montaner J, et al. Thrombolysis-related hemorrhagic infarction: a marker of early reperfusion, reduced infarct size, and improved outcome in patients with proximal middle cerebral artery occlusion. Stroke 2002;33:1551-6.

26. Kirchhof K, Welzel T, Mecke C, et al. Differentiation of white, mixed, and red thrombi: value of CT in estimation of the prognosis of thrombolysis phantom study. Radiology 2003;228:126-30.

27. Rubiera M, Ribo M, Delgado-Mederos R, et al. Tandem internal carotid artery/middle cerebral artery occlusion: an independent predictor of poor outcome after systemic thrombolysis. Stroke 2006;37:2301-5.

\section{Stay a step ahead with Online First}

We publish all our original articles online before they appear in a print issue. This means that the latest clinical research papers go straight from acceptance to your browser, keeping you at the cutting edge of medicine. We update the site weekly so that it remains as topical as possible. Follow the Online First link on the home page and read the latest research. 